\title{
AN EXTENSION OF ANDO-KRIEGER'S THEOREM TO ORDERED BANACH SPACES
}

\author{
V. CASELLES
}

(Communicated by John B. Conway)

\begin{abstract}
In this paper it is shown that an operator defined on a suitable ordered Banach space of measurable functions by a positive, irreducible kernel is never quasi-nilpotent, thus giving an extension of Ando-Krieger's theorem for operators defined on ordered Banach spaces.
\end{abstract}

Roughly speaking, Ando-Krieger's theorem says that positive, irreducible kernel operators on some Banach spaces of measurable functions are never quasi-nilpotent [6, V. 6.5, or 10, Theorem 136.9]. Recent extensions of Ando-Krieger's theorem in the framework of Banach lattices have been given in [3 and 5] (see also [4, 8]). It is our purpose in this short note to show that a similar result holds for kernel operators acting in more general ordered vector spaces including Sobolev spaces defined on sufficiently smooth domains of $\mathbf{R}^{n}$. For an interesting application of this kind of result see, for example, [1]. Following H. H. Schaefer [7], we always assume that the positive cone of an ordered Banach space is closed. For other definitions and terminology we refer to [7].

From now on we assume that $(X, \Sigma, \mu)$ is a finite measure space and denote by $L^{0}(X, \Sigma, \mu)$, or simply $L^{0}$, the set of all $\mu$-measurable functions on $(X, \Sigma, \mu)$. The cone of all positive $\mu$-measurable functions will be denoted by $L_{+}^{0}$. Recall that the family of set $\left\{V_{n}: n=1,2,3, \ldots\right\}$, where $V_{n}=\left\{f \in L^{0}: \mu\left(x:|f(x)| \geq n^{-1}\right) \leq\right.$ $\left.n^{-1}\right\}$ is a basis of neighborhoods of the origin for the topology of convergence in $\mu$-measure in $L^{0}$.

To state Theorem 1 below in a simple way, let us define

DEFINITION. Let $t: X \times X \rightarrow \mathbf{R}^{+}$be a positive measurable function. We say that $t$ is an irreducible kernel if $\int_{X-S} \int_{S} t(x, y) d \mu(x) d \mu(y)>0$ for all $S \in \Sigma$ such that $\mu(S)>0, \mu(X-S)>0$.

We give now our extension of Ando-Krieger's theorem. The key for the proof is H. H. Schaefer's approach to the proof of this theorem [6, V.6.5 and Lemma V.6.4].

THEOREM 1. Let $H$ be an ordered Banach space, $H \subseteq L^{0}$, with a nontrivial positive cone $C=L_{+}^{0} \cap H \neq\{0\}$. Let $T: H \rightarrow H$ be a bounded linear operator on $H$ induced by a positive, irreducible kernel $t(\cdot, \cdot)$, i.e. given by

$$
T f(y)=\int_{X} t(x, y) f(x) d \mu(x), \quad f \in H,
$$

Received by the editors December 1, 1986 and, in revised form, March 5, 1987.

1980 Mathematics Subject Classification (1985 Revision). Primary 47B55; Secondary 47A10.

Key words and phrases. Ordered Banach space, irreducible kernel, spectral radius. 
$\mu$-almost everywhere. Then the spectral radius of $T, r(T)$, is strictly positive. If $C$ is total in $H$ (i.e. $\overline{C-C}=H$ ) and $T$ is compact, then there exists a strictly positive eigenfunction associated to $r(T)$.

REMARK. Observe that there exists a function $e \in C$ which is strictly positive almost everywhere with respect to $\mu$. In fact, $C \neq\{0\}$, let $f \in C, f \neq 0$ and let

$$
e:=R(\lambda, T) f=\sum_{n=0}^{\infty} \lambda^{-n-1} T^{n} e \geq 0 .
$$

Since $C$ is closed in $H, e \in C$ and the strict positivity of $e$ follows from $\lambda e \geq T e$ and the irreducibility of the kernel of $T$. From now on $e$ denotes the function here constructed.

ProOF. We may assume without loss of generality that $\|T: H \rightarrow H\| \leq 1$. Let $D_{T}$ be the natural domain of $T$, defined by: $D_{T}:=\left\{u \in L^{0}: \int t(x, y)|u(x)| d \mu(x)<\right.$ $\infty$ a.e. $\}[9]$.

It is clear that $T$ can be extended to a linear map from $D_{T}$ into $L^{0}$. Let $Q$ be the solid hull of $C_{1}:=\left\{\mu \in C:\|u\|_{H} \leq 1\right\}$ i.e. $Q=\left\{u \in L^{0}\right.$ : there exists $v \in C_{1}$ such that $|u| \leq v\}$.

If we call the natural extension of $T$ to $D_{T}$ again by $T$, then $T(Q) \subseteq Q$. In fact,

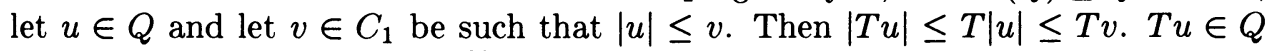
since $T v \in C_{1}$. Let $E_{Q}=\bigcup_{n=1}^{\infty} n Q$. We can introduce a norm in $E_{Q}$ using the Minkowski functional of $Q$. In fact, for $x \in E_{Q}$ define $\|x\|_{Q}: \inf \{\delta>0: x \in \delta Q\}$. The only thing to prove is that $\|x\|_{Q}>0$ for $x \in E_{Q}, x \neq 0$. To do that, observe that $Q$ is a bounded set in $L^{0}$. Otherwise, we can find a neighborhood of the origin in $L^{0}$, call it $V$, and a sequence $x_{n} \in C_{1}$ such that $x_{n} \notin 2^{n} n V$. Let $z=\sum_{n=1}^{\infty} 2^{-n} x_{n}$. Since $x_{n} \in C_{1}$ and $C$ is closed in $H$, it follows that $z \in C_{1} \subseteq Q$ and $z \geq 2^{-n} x_{n}$. Hence, $z \notin n V$ for all $n \in \mathbf{N}$. This contradiction proves that $Q$ is bounded in $L^{0}$. Hence, if $\|x\|_{Q}=0, x \in n^{-1} Q$ for all $n=1,2, \ldots$. Therefore $x=0$. Assume now that $r(T)=0$. Let $\lambda>0$. Using the Neumann series expansion of the resolvent, we see that $R(\lambda, T) e \geq \lambda^{-1} e$. Hence, $\left\|\lambda^{-1} e\right\|_{Q} \leq\|R(\lambda, T) e\|_{Q}$ for all $\lambda>0$. Thus, $\lim _{\lambda \rightarrow 0^{+}}\|R(\lambda, T) e\|_{Q}=+\infty$. Let $\lambda_{n} \rightarrow 0+$ be such that $R\left(\lambda_{n}, T\right) e \notin n Q$ for each $n=1,2, \ldots$ Thus, $\left\|R\left(\lambda_{n}, T\right) e\right\|_{H} \geq n$ for $n=1,2, \ldots$. We have found a sequence $\lambda_{n} \rightarrow 0+$ such that $\left\|R\left(\lambda_{n}, T\right) e\right\|_{H} \rightarrow \infty$. Let $z_{n}:=q_{n} R\left(\lambda_{n}, T\right) e$ where $q_{n}:=2^{-n}\left\|R\left(\lambda_{n}, T\right) e\right\|_{H}^{-1}$. Let $z:=\sum_{k=1}^{\infty} z_{k}$. Since $C$ is closed in $H, z_{n}, z \in C$. Let $y_{n}:=\sum_{k=n}^{\infty} z_{k}, n=1,2, \ldots$. Since for all $k \geq n,\left(\lambda_{n}-T\right) z_{k}=\left(\lambda_{n}-\lambda_{k}\right) z_{k}+q_{n} e$, it follows that $T y_{n} \leq \lambda_{n} y_{n}, n=1,2, \ldots$ Let $I_{z}:=\left\{u \in L^{0}:|u| \leq n z\right.$ for some integer $n \geq 1$. Let $u \in I_{z}$ and let $n \geq 1$ be such that $|u| \leq n z$. Then

$$
\int_{X} t(x, y)|u(x)| d \mu(x) \leq n \int_{X} t(x, y) z(x) d \mu(x) \leq n T z(y) \leq n \lambda_{1} z(y) .
$$

Define $T_{0}: I_{z} \rightarrow I_{z}$ by

$$
T_{0} u(y)=\int t(x, y) u(x) d \mu(x), \quad u \in I_{z} .
$$

We show that $r\left(T_{0}\right)=0$. For that, observe that for all $n=1,2, \ldots, y_{n}$ is a order unit in $I_{z}$ (i.e. for each $n \in \mathbf{N}$ we can find $k_{n}>0$ such that $\left.y_{n} \leq z \leq k_{n} y_{n}\right)$ and $T_{0} y_{n} \leq \lambda_{n} y_{n}$. Hence $r\left(T_{0}\right) \leq \lambda_{n}$, i.e. $r\left(T_{0}\right)=0$. 
It is easy to see that $I_{z}$ can be identified with the dual of the AL-space $L^{1}(X, \Sigma, z d \mu)$ and $T_{0}$ coincides with the adjoint of the operator $S_{0}$ on $L^{1}(X, \Sigma, z d \mu)$ given by

$$
S_{0} v(x)=\int t(x, y) v(y) z(y) d \mu(y)
$$

$v \in L^{1}(X, \Sigma, z d \mu)$. Since $z(y)>0$ a.e. and $t(\cdot, \cdot)$ is an irreducible kernel, $t(x, y) z(y)$ is also a positive, irreducible kernel. Hence, $S_{0}$ is a positive irreducible kernel operator on $L^{1}(X, \Sigma, z d \mu)[10,136.3]$. By Ando-Krieger's theorem [6, V.6.5, or 10, 136.9] $r\left(S_{0}\right)>0$. Therefore $r\left(T_{0}\right)>0$. A contradiction. Therefore, $r(T)>0$. The last assertion is a consequence of Krein-Rutman's theorem [7, Corollary to 2.4 Appendix]. Remark that any positive eigenfunction $u \in C$ associated to $r(T)$ must be strictly positive a.e. because $t(\cdot, \cdot)$ is an irreducible kernel.

REMARK. We have not been able to prove in Theorem 1 the uniqueness of the positive eigenfunction associated to $r(T)$. This could be done with supplementary information about the cone $C$ or $T$, available in some concrete problems. This is the case, for example, in [1].

ACKNOWLEDGMENT. I gratefully acknowledge a grant from the "Ministerio de Educación y Ciencia de España." This paper was written when I was at the University of Tübingen during the academic year 1986-87. I would like to thank the AG Funktional analysis of this University and especially Professor H. H. Schaefer and Professor R. Nagel for their hospitality. I am also indebted to Professor M. Zerner (University of Nice) for a stimulating discussion and to Professor A. Marquina (University of Valencia) for his constant help and advice.

\section{REFERENCES}

1. T. Ando and M. Zerner, Sur une valeur propre d'un opérateur, Comment. Math. Phys. 93 (1984), 123-139.

2. H. Amann, Fixed point equations and nonlinear eigenvalue problems in ordered Banach spaces, SIAM Rev. 18 (1976), 620-709.

3. V. Caselles, On irreducible operators on Banach lattices, Indag. Math. 89 (1986), 11-16.

4. J. J. Grobler, Band irreducible operators, Indag. Math. 89 (1988), 405-409.

5. B. de Pagter, Irreducible compact operators, Math. Z. 192 (1986), 149-153.

6. H. H. Schaefer, Banach lattices and positive operators, Springer-Verlag, Berlin, Heidelberg and New York, 1974.

7. __ Topological vector spaces, Macmillan, 1966.

8. __ On theorems of de Pagter and Ando-Krieger, Math. Z. 192 (1986), 155-157.

9. P. Szeptycki, Notes on integral transformations, Dissertationes Math. 231 (1984).

10. A. C. Zaanen, Riesz spaces. II, North-Holland, 1983.

11. M. Zerner, Positive operators in Reggeon field theory, why?, Aspects of Positivity in Functional Analysis (R. Nagel, U. Schlotterback, and M. P. H. Wolff, eds.), North-Holland, 1986, pp. 271-275. SPAIN

Department of Mathematics, University of Valencia, Burjasot (Valencia),

Current address: c/o Dr. Moliner, 50 Burjasot (Valencia), Spain 